\title{
Deutschland und die Finanzkrise(n)
}

Vor zehn Jahren schwappte die Krise der US-amerikanischen Hypothekenverbriefungen nach Europa über. Seither leben wir im Krisenmodus. Der von den US-Immobilienmärkten ausgehenden internationalen Finanzkrise (2007 bis 2009) folgten die irische Immobilien- und Bankenkrise (2008 bis 2010), die spanische Immobilien- und Bankenkrise (2010 bis 2012), die südeuropäischen Staatsschuldenkrisen (ab 2010), die Krise der Seeschifffahrt (ab 2008) und der auf Schiffsfinanzierungen spezialisierten Banken (ab 2013), die Turbulenzen des europäischen Finanzsystems im zweiten Halbjahr 2011 und die Krisen der Unternehmenskredite griechischer und italienischer Banken (seit 2012). All diese Krisen gingen auf eine übermäßige Kreditvergabe in den Jahren vor 2008 zurück.

Für die deutschen Steuerzahler waren die Krisen sehr teuer: Westdeutsche Landesbank (WestLB) 18 Mrd. Euro, Industriekreditbank (IKB) 9,6 Mrd. Euro, Hypo Real Estate (HRE) 14 Mrd. Euro, HSH Nordbank 16 Mrd. Euro, Sächsische Landesbank mindestens 1,4 Mrd. Euro, Landesbank Baden-Württemberg 5 Mrd. Euro, BayernLB 10 Mrd. Euro, Commerzbank 3 bis 6 Mrd. Euro - zusammen kommt das auf deutlich mehr als 70 Mrd. Euro. Dabei sind die Verluste bei BremenLB, NordLB und KfW IPEX noch nicht berücksichtigt. Ähnlich hohe Kosten für die Steuerzahler gab es sonst nur in Ländern, die selbst im Zentrum von Krisen standen. Dabei profitierten die deutschen Banken auch von den staatlichen Rettungsmaßnahmen in den USA, in Irland und Spanien. Und die staatliche Hilfe für Griechenland bot innen die Möglichkeit, griechische Schuldtitel rechtzeitig an griechische oder zypriotische Banken zu verkaufen, die später dann ihrerseits Staatshilfen brauchten.

Für die USA gibt es einen sehr gründlichen Bericht über Krisenursachen und Krisenverlauf durch eine vom Kongress eingerichtete unabhängige Kommission. Für Deutschland gibt es nichts Vergleichbares. Der 2009 im Vorfeld der Bundestagswahl tätige Untersuchungsausschuss des Bundestags zu Hypo Real Estate bezeichnet die Krise pauschal als Ergebnis von Fehlentwicklungen in den USA, denen Deutschland zum Opfer fiel, zunächst bei den Hypothekenverbriefungen und dann in der allgemeinen Panik nach dem Lehman-Konkurs. Warum Deutschland von diesen Fehlentwicklungen stärker betroffen war als andere Länder, wird nicht untersucht, auch nicht, warum die Verantwortlichen nicht früher gegengesteuert haben. Dabei sind spezifisch deutsche Fehlentwicklungen klar auszumachen. So verschärfte die mit dem Pfandbriefgesetz von 2005 verbundene Zugangserleichterung die Intensität des Wettbewerbs unter den Pfandbriefbanken mit der Folge, dass man bei der Finanzierung der für Pfandbriefe erforderlichen Überdeckung, also des Überschusses der als Sicherheiten dienenden Wertpapiere über den jeweiligen Nennwert des Pfandbriefs, extreme Fristentransformation betreiben musste, wenn man überhaupt im Markt überleben wollte. Die Hypo Real Estate nutzte dazu den Geldmarkt und war daher unmittelbar vom Zusammenbruch der Geldmärkte im September 2008 betroffen. Der HRE-Untersuchungsausschuss hat das Problem benannt, ohne auf die Ursachen und einen etwaigen Reformbedarf einzugehen.

Ferner ist bemerkenswert, dass die deutschen öffentlichen Banken, vor allem die Landesbanken, besonders von der Krise der Hypothekenverbriefungen in den USA und der Krise der Schiffsfinanzierungen betroffen waren und sind. Welche Rolle spielte die Governance dieser Institute? Etwa für den Mangel an Due Diligence beim Engagement der BayernLB bei der österreichischen Hypo Group Alpe Adria? Welche Rolle spielte es, dass die Wettbewerbsintensität in den für die Landesbanken zugänglichen

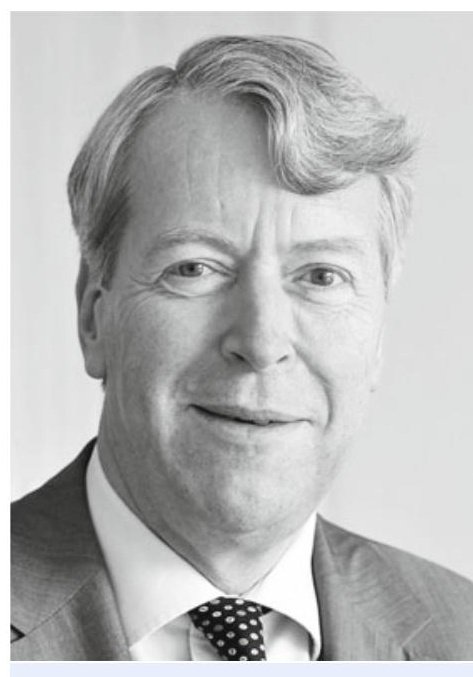

Prof. Dr. Martin Hellwig ist Direktor am Max-Planck-Institut zur Erforschung von Gemeinschaftsgütern in Bonn. 
Geschäftsfeldern sich in den 1990er Jahren noch einmal erhöht hatte? Oder dass die Landesbanken in der Übergangszeit von 2001 bis 2005 vor dem Auslaufen der staatlichen Gewährträgerhaftung noch einmal Schulden für mehr als 100 Mrd. Euro aufgenommen hatten und die Mittel dann anlegen mussten? Auch wären die Verluste bei IKB, Sachsen LB und WestLB kaum so groß geworden, wenn diese Institute nicht große Bestände an Verbriefungen in Zweckgesellschaften ausgelagert hätten, ohne sie mit Eigenkapital zu unterlegen. Im Vorfeld der Krise hatte das Bundesministerium der Finanzen die Entwicklung von Verbriefungen und von Zweckgesellschaften begrüßt und gefördert. Dabei sagt schon der gesunde Menschenverstand, dass die Aussicht auf eine vollständige Übertragung von Kreditrisiken durch Verbriefung die Anreize zur Kreditwürdigkeitsprüfung mindert und daher die Kreditrisiken erhöht, auch dass die Auslagerung riskanter Anlagen in Zweckgesellschaften die betreffenden Banken erheblichen Liquiditäts- und Solvenzrisiken aussetzen musste.

Vor 2007 sind die großen Banken stark gewachsen, in Europa noch deutlich mehr als in den USA, auch deutlich mehr als die Indikatoren für Wirtschaftstätigkeit oder Vermögen. Das Wachstum wurde überwiegend durch Schulden finanziert. Im Median der 20 größten europäischen Banken lag das Eigenkapital 1998 noch bei 6\% der Bilanzsumme, 2007 bei 3\%. Bei der Deutschen Bank unterschritt es 2007 sogar 2\%. Die zusätzliche Verschuldung kam zu großen Teilen aus Geldmarktkrediten und Derivaten und war entsprechend fragil. In der Krise bewirkte die extrem hohe Verschuldung, dass schon bald die Solvenz großer Banken in Zweifel gezogen wurde und die Refinanzierung über den Geldmarkt ins Stocken kam. Bei Lehman Brothers war das der Grund für den Konkurs. Dieser löste einen Run auf die Geldmarktfonds aus, die ihrerseits ihre Mittel von den Banken abzogen. Der Zusammenbruch der Geldmärkte im September 2009 war ein Ergebnis von klar erkennbaren Wirkungszusammenhängen, die später auch die Turbulenzen von 2011 prägten und auch heute noch systemische Risiken begründen.

Auch hier hätte es nahegelegen, die Mitverantwortung der Behörden zu prüfen. Die Eigenmittelquoten konnten so stark zurückgehen, weil die Banken ab 1996 die für die Regulierung maßgeblichen Einschätzungen der Marktrisiken von Anlagen im Handelsbuch anhand ihrer eigenen Modelle selbst bestimmen konnten und viele Risiken ausblendeten. Zur Abhängigkeit der Refinanzierung vom Geldmarkt beteuerten die Vertreter der Aufsicht im HRE-Untersuchungsausschuss ihr Unbehagen; inwiefern sie das auch im Vorhinein äußerten, ist nicht bekannt. Eine öffentliche Diskussion hat die Bundesregierung verweigert. Als z.B. im Herbst 2010 die Fraktion Bündnis 90/Die Grünen im Bundestag wissen wollte, wie oft die Vertreter der Aufsicht und der Bundesbank von ihrem Rederecht in Gremien der Banken Gebrauch gemacht hätten, um Unbehagen zu äußern, antwortete die Bundesregierung, das könne man nicht sagen, denn von den Sitzungen gebe es keine Wortprotokolle. Man kann sich kaum vorstellen, dass die Erinnerung an die Wahrnehmung des Rederechts am Fehlen von Wortprotokollen scheitert; so etwas wird behördenintern im Vorhinein vorbereitet und dokumentiert. Zu anderen Fragen hieß es, eine Veröffentlichung der gewünschten Information könne die betroffenen Banken oder gar das gesamte Finanzsystem gefährden - dies wohlgemerkt bei Banken, die schon unter der Kuratel des Bundes standen!

Auch die Reformmaßnahmen seit 2009 wurden weitgehend der öffentlichen Diskussion entzogen. Einige, wie das Bankenrestrukturierungsgesetz von 2010, konnten erkennbar nicht das leisten, was versprochen wurde, und mussten alsbald ergänzt werden. Und derzeit diskutieren wir über neue „Bad Banks“ für die faulen Kredite in den Büchern europäischer, auch deutscher, Banken. Die Risiken wird wieder der Steuerzahler tragen. Und viele der Mechanismen, die das System 2008 in den Abgrund zu stürzen drohten, sind nach wie vor virulent. Der Mangel an Transparenz und Verantwortlichkeit ist mit ein 